\title{
考虑心墙不同破坏模式的黏土心墙坝漫顶溃坝 过程数学模型
}

钟启明 ${ }^{1,2 *}$, 陈生水 ${ }^{1,2}$, 曹伟 ${ }^{1}$, 梅世昂 ${ }^{1}$

1. 南京水利科学研究院, 南京 210029 ;

2. 水利部土石坝破坏机理与防控技术重点实验室, 南京 210029

*E-mail: qmzhong@nhri.cn

收稿日期: 2017-05-07; 接受日期: 2017-06-07; 网络出版日期: 2017-08-10

国家自然科学基金(51379129,51539006, 51509164)和江苏省自然科学基金(BK20161121, BK20150077)资助项目

\begin{abstract}
摘要基于黏土心墙坝的漫顶溃决机理, 提出一个模拟其溃决过程的数学模型. 该模型基于坝体形状和漫顶 水流特征确定下游坡冲蚀时的初始冲坑位置, 采用宽顶堰流量公式计算溃口流量, 并利用溯源冲刷公式模拟坝 壳料的冲蚀; 通过力矩平衡法与力学平衡法分别模拟了心墙的倾倒破坏与剪切破坏; 为了模拟实际溃坝情况, 模型考虑了坝体的单侧冲蚀、两侧冲蚀及坝基的冲蚀; 采用按时间步长迭代的数值计算方法模拟溃坝时的水 土耦合过程. 选择国内外 10 个具有实测资料的心墙坝漫顶溃坝案例对模型进行验证, 通过比对峰值流量、溃口 最终宽度及溃坝历时等参数发现, 模型计算结果与实测值的相对误差可满足工程需求, 验证了模型的合理性.
\end{abstract}

关键词 黏土心墙坝, 漫顶, 心墙破坏, 数学模型

\section{1 引言}

我国水利水电工程建设正经历一个前所未有的快 速发展时期, 一大批水工建筑物, 尤其是枢纽工程中最 重要的挡水建筑物——大坝, 超越了以往的规模 ${ }^{[1]}$. 在 已建成的水库大坝中, 黏土心墙坝是一种常用的坝型, 它使用透水性较好的砂或砂砾做坝壳, 以防渗性较好 的黏性土作为防渗体设在坝的剖面中心位置. 这种坝 型与一般土坝相比, 具有抗滑、抗渗稳定性好、施工 方便、坝体经济等特点, 适应在深厚覆盖层地基及复 杂地质条件下填筑, 是一种极具竞争力的坝型 ${ }^{[2]}$. 值得 注意的是, 黏土心墙坝在给人类社会带来巨大经济效
益的同时也存在着溃坝的风险. 据水利部大坝安全管 理中心统计 ${ }^{[3]}, 1954$ 2014年, 我国共有3530座水库大 坝溃决, 漫顶溃坝数占总数的 $50 \%$ 以上, 其中共有 183 座黏土心墙坝发生漫顶溃坝, 约占溃坝总数的 $5.2 \%$. 1963 年 8 月发生在河北的“63.8”洪水, 导致2座中型水 库(刘家台水库、佐村水库)坝高分别为 35.8 和 $35.0 \mathrm{~m}$ 的黏土心墙坝发生漫顶溃决, 造成948人死亡和大量财 产损失 ${ }^{[4]} ; 1975$ 年8月发生在河南的“75.8”洪水, 导致板 桥水库坝高 $24.5 \mathrm{~m}$ 的黏土心墙坝发生漫顶溃决(图1), 造成 26000 余人死亡和大量财产损失及生态环境严重 破坏 ${ }^{[4,5]}$. 因此有必要深入研究黏土心墙坝漫顶溃决机 理, 揭示水土耦合条件下黏土心墙坝的破坏规律及破

引用格式: 钟启明, 陈生水, 曹伟, 等. 考虑心墙不同破坏模式的黏土心墙坝漫顶溃坝过程数学模型. 中国科学: 技术科学, 2017, 47: 992-1000 Zhong Q M, Chen S S, Cao W, et al. Numerical model for clay core dam breach process due to overtopping considering different failure modes of core wall (in Chinese). Sci Sin Tech, 2017, 47: 992-1000, doi: 10.1360/N092017-00135 


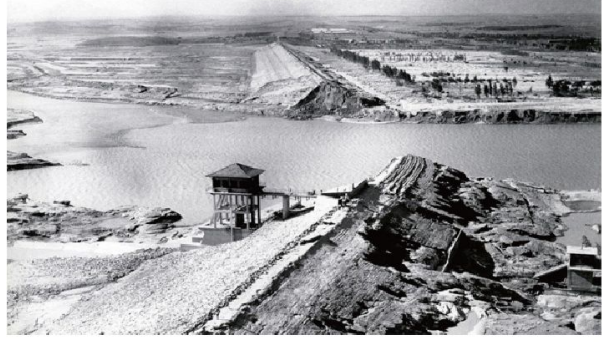

图 1 “75.8”洪水后板桥水库黏土心墙坝最终溃口

坏过程, 建立能正确揭示黏土心墙坝漫顶溃决机理, 合理模拟漫顶溃坝过程的数学模型, 提升黏土心墙坝 漫顶溃决时溃口发展过程和溃坝洪水流量过程的预 测精度, 进而为溃坝洪水风险分析和应急预案的编制 提供理论和技术支撑。

\section{2 黏土心墙坝漫顶溃决机理}

对于均质土坝漫顶溃决问题, 国内外学者开展了 大量不同比尺的物理模型试验研究, 提出了一系列的 数学模型 ${ }^{[5-8]}$. 对于黏土心墙坝, 由于模型试验的复杂 性, 目前仅欧盟IMPACT 项目 ${ }^{[9]}$ 于 2003 年开展的坝高 $6.0 \mathrm{~m}$ 的冰碛土心墙堆石坝野外漫顶溃决试验(图2)和 南京水利科学研究院陈生水等人 ${ }^{[10]}$ 利用土石坝溃坝离 心模型试验系统于 2010 年开展的原型坝高 $16.0 \mathrm{~m}$ 的黏 土心墙堆石坝漫顶溃决离心模型试验(图3). 通过模型 试验可以发现: 在漫顶水流的作用下,坝壳料的冲蚀 基本上以表层冲蚀为主, 首先在下游坡出现初始冲坑, 随着下游坝壳冲蚀程度的加剧, 心墙下游侧面逐渐暴 露临空, 在上游水压力和土压力的共同作用下, 心墙发 生倾倒破坏或剪断破坏; 心墙破坏后, 由于漫顶水头 突然增加, 冲蚀将进一步加剧, 溃口流量迅速增加, 随 着库水位的下降, 溃口流量也将逐渐下降至溃坝结束.

基于黏性土墙坝的漫顶溃决机理,建立了一个黏 土心墙坝漫顶溃坝过程数学模型. 模型将漫顶溃坝过 程分为 3 个阶段: (1) 下游坝坡出现初始冲坑, 冲坑的 位置由坝体形状和漫顶水头等条件确定, 在漫顶水流 的作用下冲坑处边坡逐渐变陡, 随后向上游发生溯源 冲刷, 模型假设下游坡坝壳料在水流作用下发生溯源 冲刷时边坡保持内摩擦角(图4, (1) (3)); (2) 随着顶部 和下游坡坝壳料的不断冲刷, 心墙逐渐暴露, 在上游 水压力和土压力的作用下, 心墙发生倾倒破坏或剪切

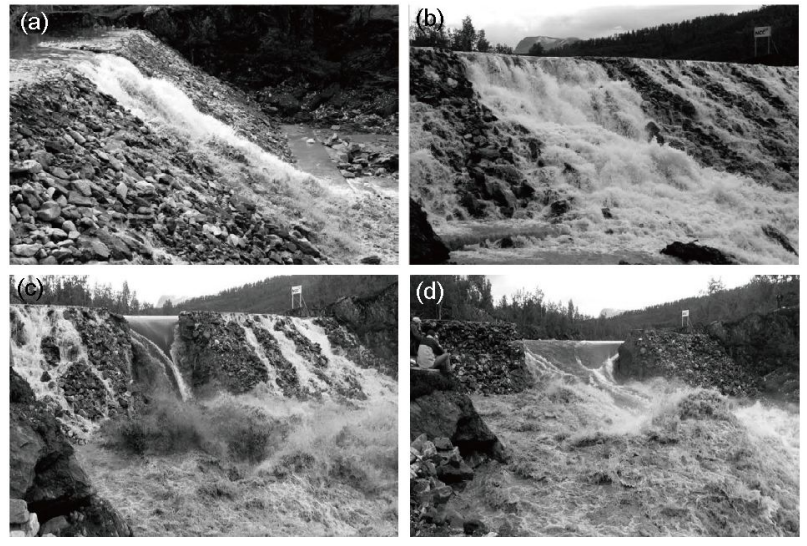

图 2 冰碛土心墙堆石坝漫顶溃决过程现场模型试验. (a) 下游坡发生冲蚀; (b) 溃口进一步扩展; (c) 心墙发生破坏; (d) 溃口继续加深展宽
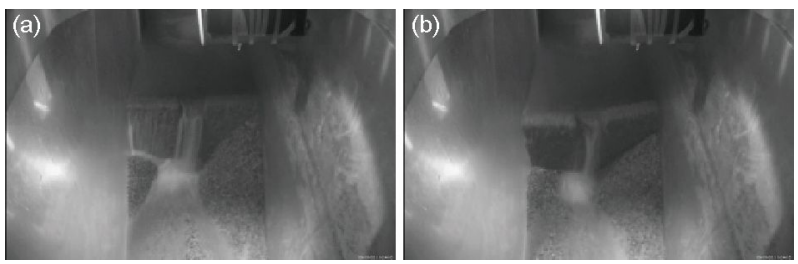

图 3 黏土心墙坝漫顶溃决过程离心模型试验. (a) 下游坝 壳冲蚀心墙暴露; (b) 心墙发生破坏

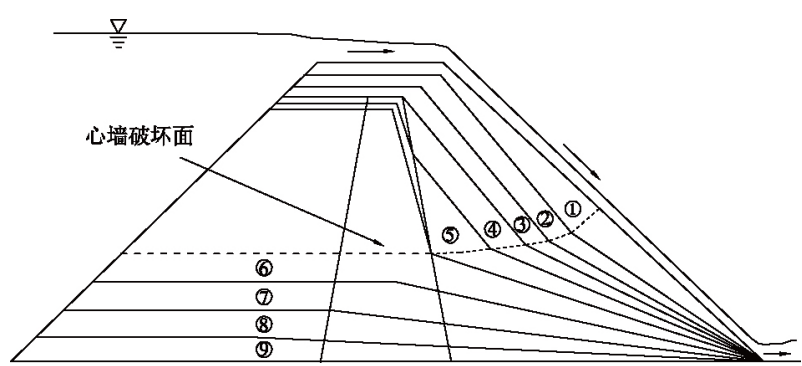

图 4 黏土心墙坝漫顶溃坝过程示意图

破坏(图4, (4) (6)); (3) 心墙倒塌后, 溃口在漫顶水流的 作用下不断增大, 当库水下泄完毕或者溃口水流无法 继续冲蚀土体后, 溃坝过程结束(图4, (7) (9)). 具体的 模拟过程如下文.

\section{3 黏土心墙坝漫顶溃坝过程数学模型}

\section{1 水量平衡与库水位变化关系}

大坝漫顶溃决过程中, 上游库水位是一个动态变 
化的过程, 包括上游河道的入流和溃口出流. 在计算 上游库水高程变化时, 需同时考虑入库流量, 溃口出 流量, 溢洪道和闸门下泄流量, 整个过程服从水量平 衡方程:

$A_{s} \frac{\mathrm{d} z_{s}}{\mathrm{~d} t}=Q_{\text {in }}-Q_{b}-Q_{\text {spill }}-Q_{\text {sluice }}$,

式中, $t$ 为时间; $z_{s}$ 为库水位; $A_{s}$ 为水库库面面积; $Q_{\text {in }}$ 为入 库流量; $Q_{b}$ 为溃口出流量; $Q_{\text {spill }}$ 为溢洪道出流量; $Q_{\text {sluice }}$ 为闸门下泄流量.

\section{2 溃口流量过程}

室内试验与现场观测研究结果表明, 流经溃口的 流量可以采用宽顶堰流公式进行计算, 溃口流量可用 下式表示:

$Q_{b}=k_{\mathrm{sm}}\left(c_{1} b H^{1.5}+c_{2} m H^{2.5}\right)$,

式中, $b$ 为溃口底宽; $H$ 为溃口处水深, $H=z_{s}-z_{b}$, 其中 $z_{b}$ 为溃口底部高程; $m$ 为溃口边坡坡比(水平/垂直); $c_{1}, c_{2}$ 为修正系数, 本文中 $c_{1}=1.7 \mathrm{~m}^{0.5} / \mathrm{s}, c_{2}=1.1 \mathrm{~m}^{0.5} / \mathrm{s}^{[11]} ; k_{\mathrm{sm}}$ 为 尾水淹没修正系数 ${ }^{[12]}$, 可由下式计算:

$k_{\mathrm{sm}}=\left\{\begin{array}{cc}1.0 & \frac{z_{t}-z_{b}}{z_{s}-z_{b}}<0.67, \\ 1.0-27.8\left(\frac{z_{t}-z_{b}}{z_{s}-z_{b}}-0.67\right)^{3} & \text { 其他, }\end{array}\right.$

式中, $z_{t}$ 为尾水高度.

\section{3 初始冲坑的位置}

荷兰学者Visser ${ }^{[13]}$ 指出, 漫顶水流流经下游坝坡 流速逐渐增大并趋于定值, 本文将此流速最大的位置 定义为初始冲坑所在, 流速达到定值对应的坝坡长度 $l_{n}$ 可表示为

$l_{n}=\frac{2.5\left(F r_{n}^{2}-1\right) d_{n}}{\tan \beta}$,

式中, $\beta$ 为下游坝坡的坡角; $d_{n}$ 为下游坝坡上的水流深 度, 其中 $n$ 表示法向方向; $F r_{n}$ 为弗劳德数, 可表示为

$F r_{n}^{2}=\frac{U_{n}^{2}\left(B_{t}\right)_{n}}{\mathrm{~g} d_{n} B_{n} \cos \beta}=\frac{U_{n}^{2}}{\mathrm{~g} d_{n} \cos \beta}$,

式中, $U_{n}$ 为断面平均流速; $B_{t}$ 为溃口顶宽; $B_{n}$ 为溃口沿 着下游坡的宽度, 假设 $B_{t}=B_{n} ; \mathrm{g}$ 为重力加速度; $U_{n}$ 和 $d_{n}$
可分别表示为

$U_{n}=C \sqrt{R_{n} \sin \beta}=C \sqrt{H \sin \beta}\left(x \geq l_{n}\right)$,

$d_{n}=\frac{Q_{b}}{U_{n} B_{n}}\left(x \geq l_{n}\right)$,

式中, $R_{n}$ 为溃口处的水力半径, 假设初始溃口为矩形, 因此, $R_{n}=H=z_{s}-z_{b}$, 其中 $z_{s}$ 为库水位; $z_{b}$ 为溃口底部高程; $Q_{b}$ 为溃口流量; $C$ 为谢才系数, 可由下式获得:

$C=\frac{1}{n} R_{n}^{\frac{1}{6}}=\frac{1}{n} H^{\frac{1}{6}}$,

式中, $n$ 为曼宁系数, 可通过下式表达:

$n=d_{50}^{\frac{1}{6}} / A_{n}$,

式中, $A_{n}$ 为经验系数, 本文选取 $12^{[14]}$.

\section{4 溃口发展}

初始冲坑形成后, 在溃口水流的作用下, 坝顶和下 游坡均遭受冲蚀, 初始冲坑 $\left(x=l_{n}\right)$ 处的边坡逐渐变陡, 下游坝坡坡角由初始坡角 $\beta\left(t=t_{0}\right)$ 逐渐变为坝壳料内摩 擦角 $\varphi_{1}\left(t=t_{c}\right)$ (图5).

坝壳料的冲蚀率可采用下式计算 ${ }^{[15]}$ :

$E=k_{d}\left(\tau_{b}-\tau_{c}\right)$,

式中, $E$ 为冲蚀率; $k_{d}$ 为冲蚀系数, 常通过试验量测 ${ }^{[16,17]}$ 或采用经验公式 ${ }^{[18]}$ 求取; $\tau_{b}$ 为水流剪应力; $\tau_{c}$ 为坝料临 界剪应力, 可通过希尔兹曲线确定 ${ }^{[19]}$. 其中, $\tau_{b}$ 与 $k_{d}$ 计 算公式如下:

$\tau_{b}=\frac{\rho_{w} g n^{2} Q_{b}^{2}}{A_{w}^{2} R^{\frac{1}{3}}}$,

式中, $\rho_{w}$ 为水的密度; $A_{w}$ 为水流面积.

$k_{d}=\frac{10 \rho_{w}}{\rho_{d}} \exp \left[-0.121 c \% \%^{0.406}\left(\frac{\rho_{d}}{\rho_{w}}\right)^{3.1}\right]$,

式中, $\rho_{w}$ 为水的密度; $\rho_{d}$ 为土体干密度; $c \%$ 为黏粒含量. 对于坝顶的冲蚀, 可采用式(10)模拟; 对于下游坡 的变陡过程(图5), 可采用下式表示:

$\mathrm{d} \beta=\frac{\left(E_{1}-E_{0} / \cos \beta\right) \mathrm{d} t}{l_{n}}$,

式中, $\mathrm{d} \beta$ 为下游坡坡角增加值; $E_{1}$ 为下游坡初始冲坑 


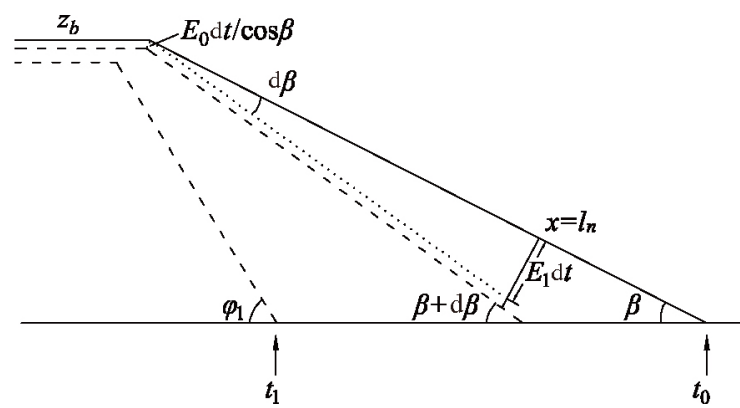

图 5 坝顶及下游坡冲蚀示意图

处冲蚀速率; $E_{0}$ 为坝顶处冲蚀率.

当下游坡坡角达到坝壳料内摩擦角 $\varphi_{1}$ 后, 在溃坝 水流作用下, 溯源冲刷继续向上游发展至黏土心墙处, 并假设下游坡保持内摩擦角. 可采用下式表示坝壳料 的溯源冲刷过程 ${ }^{[20]}$ :

$\frac{\mathrm{d} x}{\mathrm{~d} t}=C_{T} q^{1 / 3} H_{e}^{1 / 2}$,

式中, $\mathrm{d} x / \mathrm{d} t$ 为溯源冲刷速率; $C_{T}$ 为与坝料物理力学指 标相关的溯源冲刷系数, 可采用文献[21]提供的方法 获取; $q$ 为溃口单宽流量; $H_{e}$ 水流高度.

对于坝轴线处的溃口发展, 假设溃口处的坡角为 坝壳料的内摩擦角 $\varphi_{1}$, 横向与纵向扩展速度可表示为 (图6)

$\Delta B_{t}=\frac{n_{\mathrm{loc}} \Delta z_{b}}{\sin \varphi_{1}}$,

$\Delta B_{b}=n_{\mathrm{loc}} \Delta z_{b}\left(\frac{1}{\sin \varphi_{1}}-\frac{1}{\tan \varphi_{1}}\right)$,

式中, $\Delta B_{t}$ 为溃口顶宽增加值; $\Delta B_{b}$ 为溃口底宽增加值; $n_{\mathrm{loc}}$ 溃口所在位置 $\left(n_{\mathrm{loc}}=1\right.$ 表示单侧冲蚀; $n_{\mathrm{loc}}=2$ 表示两侧 冲蚀); $\Delta z_{b}$ 为溃口底部冲蚀深度增加值.

\section{5 心墙破坏}

随着坝壳料的冲蚀, 心墙逐渐暴露, 在上游水压力 和土压力的作用下, 当坝壳料冲蚀到一定程度后, 心 墙可能产生拉裂缝而导致倾覆破坏或剪切破坏(图7), 本文假设破坏的心墙两侧呈直立状. 对于心墙可能遭 受拉应力导致的倾覆, 采用力矩平衡法模拟其安全性; 对于心墙可能发生的剪切破坏, 采用力学平衡法模拟 其安全性. 在每个时间步计算比较 2 种可能破坏模式 的安全性, 从而选择出适当的破坏模式.

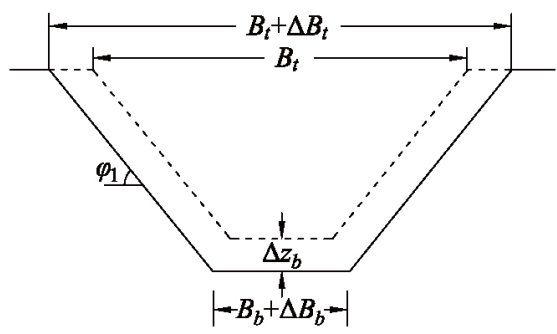

图 6 坝轴线处溃口发展过程

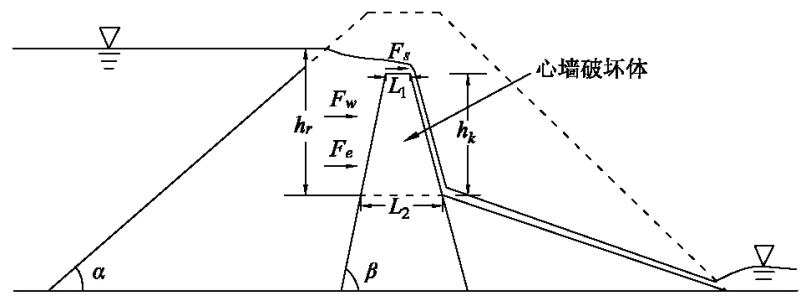

图 7 心墙破坏示意图

若心墙发生倾覆破坏, 向下游各种推力作用在心 墙破坏面上的力矩 $M_{o}$ 可表示为

$$
\begin{aligned}
M_{o}= & F_{s} \cdot h_{k}+F_{w} \cdot \frac{h_{k}\left(2 h_{r}-h_{k}\right)+h_{r}}{3\left(h_{r}-h_{k}+h_{r}\right)} \\
& +F_{e} \cdot \frac{h_{k}\left(2 h_{r}-h_{k}\right)+h_{r}}{3\left(h_{r}-h_{k}+h_{r}\right)},
\end{aligned}
$$

式中, $F_{s}$ 为溃口漫顶水流作用在心墙顶部的剪切力; $F_{w}$ 为库水作用在心墙上的水压力; $F_{e}$ 为上游坝壳料作用 在心墙上的土压力; $h_{k}$ 为破坏心墙的高度; $h_{r}$ 为库水位 距离心墙破坏面的高度. 其中, $F_{s}, F_{w}$ 与 $F_{e}$ 可由下式得 出:

$F_{s}=\tau_{b} \cdot B_{b} \cdot L_{1}$,

$F_{w}=\rho_{w} g \cdot B_{b} \cdot h_{k}\left(h_{r}-0.5 h_{k}\right)$,

$F_{e}=\frac{1}{2} K_{a} \cdot \gamma_{s} \cdot\left(1-p_{1}{ }^{\prime}\right) \cdot B_{b} \cdot h_{k}^{2}$,

式中, $L_{1}$ 为心墙顶部宽度; $K_{a}$ 为主动土压力系数; $\gamma_{s}$ 为 土颗粒的容重; $p_{1}{ }^{\prime}$ 为坝壳料的孔隙率.

抵抗力作用在心墙破坏面上的力矩 $M_{r}$ 可表示为

$M_{r}=2 A_{t} \cdot C_{2} \cdot \frac{h_{k}\left(2 h_{r}-h_{k}\right)+h_{r}}{3\left(h_{r}-h_{k}+h_{r}\right)}+\frac{1}{2} W \cdot L_{2}$,

式中, $A_{t}$ 为破坏心墙的截面积; $C_{2}$ 为黏土心墙的黏聚 力; $W$ 为破坏面以上心墙的重量; $L_{2}$ 为心墙破坏面宽 
度. 其中, $A_{t}$ 与 $W$ 可表示为

$A_{t}=\frac{1}{2}\left(L_{1}+L_{2}\right) \cdot h_{k}$,

$W=0.5 \gamma_{s} \cdot\left(1-p_{2}{ }^{\prime}\right)\left(L_{1}+L_{2}\right) h_{k} B_{b}$,

式中, $p_{2}{ }^{\prime}$ 为心墙料的孔隙率.

心墙发生倾覆破坏的临界条件为

$M_{o}=M_{r}$.

若心墙发生剪切破坏, 作用在心墙上的驱动力包 括水流剪切力、水压力与土压力; 作用在心墙上的抵 抗力包括心墙破坏体与两侧及底部的黏结力以及底 部摩擦力. 心墙发生剪切破坏的临界条件为

$F_{s}+F_{w}+F_{e}=F_{c}+F_{f}$,

式中, $F_{c}$ 为心墙破坏体与侧面及底部的黏结力; $F_{f}$ 为心 墙破坏体底部的摩擦力. 可分别表示为

$F_{c}=\left(2 A_{t}+L_{2} \cdot B_{b}\right) \cdot C_{2}$,

$F_{f}=W \cdot \tan \varphi_{2}$,

式中, $\varphi_{2}$ 为心墙料的内摩擦角.

\section{6 坝基冲蚀}

随着溃口冲深的增加, 溃口达到坝体底部, 模型 考虑了溃口水流对坝基冲蚀的可能性. 模型假设可冲 蚀的坝基呈水平层状分布(图8), 结合坝基材料的物理 力学性质, 采用冲蚀式(10)计算坝基的冲蚀过程. 模型 保证水量平衡关系, 并采用式(2)中的尾水淹没修正系 数考虑坝基冲蚀时尾水对于溃口流量过程的影响.

\section{7 溃口边坡稳定性}

心墙发生破坏后, 随着黏土心墙溃口的逐渐发展, 溃口边坡可能会发生失稳, 采用极限平衡法分析边坡 稳定性, 并假设破坏面为平面, 当满足下式时, 边坡失 稳(图9):

$F_{d}>F_{r}$,

式中, $F_{d}$ 为滑动力; $F_{r}$ 为抗滑力. 其中, $F_{d}$ 与 $F_{r}$ 分别表 示为

$F_{d}=W_{s} \sin \theta=\frac{1}{2} \gamma_{b} H_{s}^{2}\left(\frac{1}{\tan \theta}-\frac{1}{\tan \beta}\right) \sin \theta$,

$$
\begin{aligned}
F_{r} & =W_{s} \cos \theta \tan \varphi_{2}+\frac{C_{2} H_{s}}{\sin \theta} \\
& =\frac{1}{2} \gamma_{b} H_{s}^{2}\left(\frac{1}{\tan \theta}-\frac{1}{\tan \beta}\right) \cos \theta \tan \varphi_{2}+\frac{C_{2} H_{s}}{\sin \theta},
\end{aligned}
$$

式中, $H_{s}$ 为溃口边坡高度; $W_{s}$ 为破坏体重量; $\gamma_{b}$ 为土体 容重. 其中, $\gamma_{b}$ 可通过下式表示:

$\gamma_{b}=\left(1-p_{2}{ }^{\prime}\right) \gamma_{s}+p_{2}{ }^{\prime} \gamma_{w}$.

\section{8 数值计算方法}

采用按时间步长迭代的计算方法模拟黏土心墙 坝的漫顶溃坝过程, 通过在每个时间步长计算溃口流 量过程与溃口发展过程模拟溃坝过程中的水土耦合， 计算流程如图 10.

\section{4 模型验证}

\section{1 计算参数选取}

选择国内外 10 个具有实测资料的黏土心墙坝漫 顶溃坝案例对本文提出的模型进行验证. 10 个案例包 括 1 组欧盟IMPACT 项目的现场溃坝模型试验 ${ }^{[9,22]}$ 和 9 座国内外黏土心墙坝实际溃坝案例 ${ }^{[4,14,23,24]}$. 模型的计 算参数, 如坝的轮廓信息、土体物理力学指标、水库 特征信息等如表 1 和表 2 .

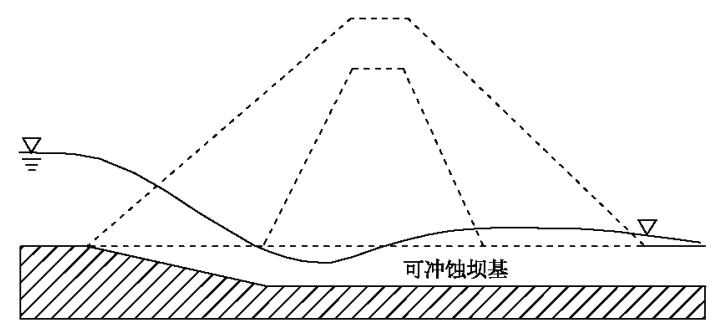

图 8 坝基冲蚀示意图

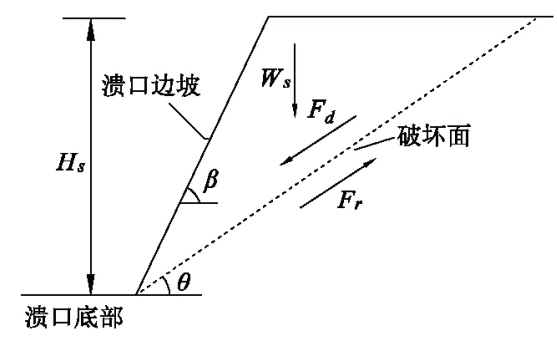

图 9 溃口边坡稳定性分析 


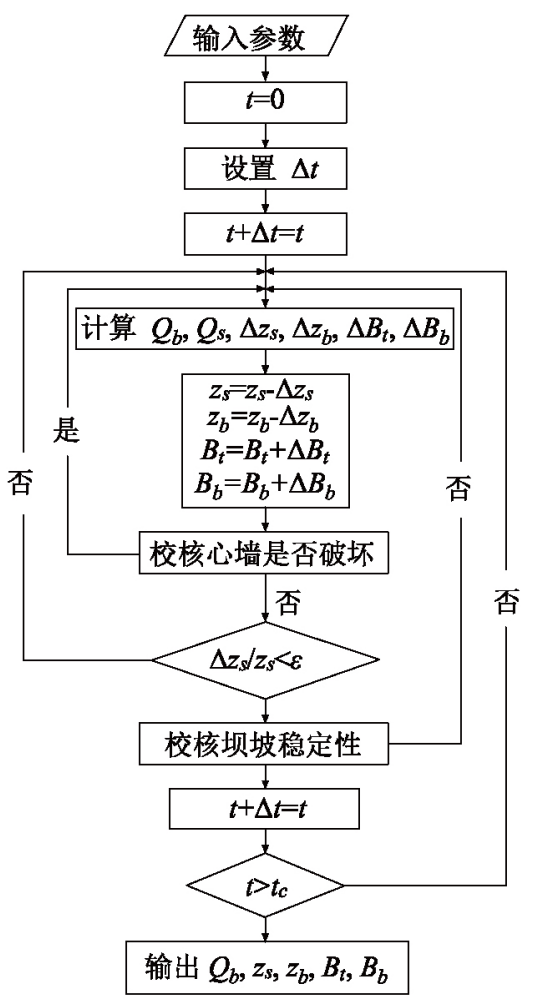

图 10 黏土心墙坝漫顶溃坝过程计算流程图

\section{2 计算结果分析}

表3列举了 10 个溃坝案例的模型计算计算结果与 实测结果, 比较的模型参数主要包括: 溃口峰值流量 $\left(Q_{p}\right)$, 溃口最终顶宽 $\left(B_{t}\right)$, 溃口最终底宽 $\left(B_{b}\right)$, 峰值流量 出现时间 $\left(T_{p}\right)$ 及溃坝历时 $\left(T_{f}\right)$.

为了更好地定量研究模型的计算误差, 采用相对 误差和均方根相对误差衡量 10 个溃坝案例模型计算
结果与实测值之间的差异. 相对误差衡量单个案例的 误差, 均方根相对误差衡量所有案例的整体误差.

相对误差可表示为

$\delta=\frac{A_{i, \text { calculated }}-A_{i, \text { measured }}}{A_{i, \text { calculated }}} \times 100 \%$,

式中, $\delta$ 为相对误差; $A_{i, \text { calculated }}$ 为参数 $A_{i}$ 的计算值; $A_{i, \text { measured }}$ 为参数 $A_{i}$ 的实测值.

均方根相对误差可表示为

$E_{\mathrm{rms}}=\sqrt{\frac{1}{N} \sum_{i=1}^{N}\left[\log \left(\frac{A_{i, \text { calculated }}}{A_{i, \text { measured }}}\right)\right]^{2}}$,

式中, $E_{\mathrm{rms}}$ 为均方根相对误差; $N$ 为案例数.

表4给出了各参数计算结果与实测结果之间的误 差情况. 主要包括各参数相对误差 $\pm 25 \%$ 以内和 $\pm 50 \%$ 以内的溃坝案例占有实测值案例的比例, 以及各参数 的均方根相对误差.

由表 4 的计算结果可以得出: 对于溃口峰值流量 $\left(Q_{p}\right)$, 相对误差 $\pm 25 \%$ 和 $\pm 50 \%$ 的溃坝案例的比例均为 $100 \%$, 均方根相对误差仅 0.042 ; 对于溃口最终顶宽 $\left(B_{t}\right)$, 相对误差 $\pm 25 \%$ 和 $\pm 50 \%$ 的溃坝案例的比例分别为 $57.1 \%$ 和 $85.7 \%$, 均方根相对误差为 0.117 ; 对于溃口最 终底宽 $\left(B_{b}\right)$, 相对误差 $\pm 25 \%$ 和 $\pm 50 \%$ 的溃坝案例的比例 分别为 $57.1 \%$ 和 $100 \%$, 均方根相对误差为 0.097 ; 由于 一般溃坝案例无溃口峰值流量出现时间 $\left(T_{p}\right)$ 的记载, 本次只衡量溃坝历时 $\left(T_{f}\right)$ 的相对误差, 由表 4 看出, 该 参数相对误差 $\pm 25 \%$ 和 $\pm 50 \%$ 的溃坝案例的比例分别为 $80.0 \%$ 和 $100 \%$, 均方根相对误差为 0.082 .

表 1 黏土心墙坝漫顶溃坝案例大坝与水库信息

\begin{tabular}{cccccccccccc}
\hline 序号 & 大坝名称 & $\begin{array}{c}\text { 坝高 } \\
(\mathrm{m})\end{array}$ & $\begin{array}{c}\text { 坝长 } \\
(\mathrm{m})\end{array}$ & $\begin{array}{c}\text { 顶宽 } \\
(\mathrm{m})\end{array}$ & $\begin{array}{c}\text { 上游 } \\
\text { 坡比 }\end{array}$ & $\begin{array}{c}\text { 下游 } \\
\text { 坡比 }\end{array}$ & $\begin{array}{c}\text { 库容 } \\
\left(\mathrm{m}^{3}\right)\end{array}$ & $\begin{array}{c}\text { 库面面 } \\
\text { 积 }\left(\mathrm{m}^{2}\right)\end{array}$ & $\begin{array}{c}\text { 初始库水 } \\
\text { 位 }(\mathrm{m})\end{array}$ & $\begin{array}{c}\text { 入流量 } \\
\left(\mathrm{m}^{3} / \mathrm{s}\right)\end{array}$ & $\begin{array}{c}\text { 坝基冲 } \\
\text { 蚀 }(\mathrm{m})\end{array}$ \\
\hline 1 & 板桥 & 24.5 & 500.0 & 8.0 & 0.384 & 0.5 & $4.92 \times 10^{8}$ & $A_{s}-h$ & 26.0 & $Q_{i n}-t$ & -5.0 \\
2 & Castlewood & 21.3 & 182.9 & 4.9 & 0.333 & 1.0 & $4.23 \times 10^{6}$ & $A_{s}-h$ & 21.6 & - & 0.0 \\
3 & Coedty & 11.0 & 262.0 & 3.1 & 0.333 & 0.333 & $3.10 \times 10^{5}$ & - & 11.1 & - & 0.0 \\
4 & Elk City & 9.1 & 564.0 & 4.9 & 0.4 & 0.4 & $7.40 \times 10^{5}$ & - & 9.4 & - & 0.0 \\
5 & Grand Rapids & 7.6 & 441.0 & 3.7 & 0.4 & 0.4 & $2.20 \times 10^{5}$ & - & 7.7 & - & 0.0 \\
6 & IMPACT现场试验 & 5.9 & 60.0 & 3.0 & 0.645 & 0.645 & $6.30 \times 10^{4}$ & $A_{s}-h$ & 5.4 & $Q_{i n}-t$ & 0.0 \\
7 & 刘家台 & 35.8 & 295.0 & 5.0 & 0.333 & 0.333 & $4.05 \times 10^{7}$ & - & 35.9 & $Q_{i n}-t$ & 0.0 \\
8 & Oros & 35.4 & 620.0 & 5.0 & 0.276 & 0.24 & $6.60 \times 10^{8}$ & $A_{s}-h$ & 35.8 & - & -4.0 \\
9 & 竹沟 & 23.5 & 300 & 5.0 & 0.4 & 0.4 & $1.54 \times 10^{7}$ & - & 23.5 & $Q_{i n}-t$ & 0.0 \\
10 & 佐村 & 35.0 & 640 & 7.0 & 0.5 & 0.5 & $4.00 \times 10^{7}$ & - & 40.0 & $Q_{i n}-t$ & 0.0 \\
\hline
\end{tabular}


钟启明等: 考虑心墙不同破坏模式的黏土心墙坝漫顶溃坝过程数学模型

表 2 黏土心墙坝漫顶溃坝案例心墙与坝壳物理力学指标

\begin{tabular}{|c|c|c|c|c|c|c|c|c|c|c|c|c|c|c|}
\hline \multirow{2}{*}{$\begin{array}{l}\text { 序 } \\
\text { 号 }\end{array}$} & \multirow[b]{2}{*}{ 大坝名称 } & \multicolumn{9}{|c|}{ 心墙 } & \multicolumn{4}{|c|}{ 坝壳 } \\
\hline & & 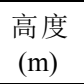 & $\begin{array}{l}\text { 顶宽 } \\
(\mathrm{m})\end{array}$ & $\begin{array}{c}\text { 上游坡 } \\
\text { 比 }\end{array}$ & $\begin{array}{c}\text { 下游坡 } \\
\text { 比 }\end{array}$ & $\begin{array}{c}d_{50} \\
(\mathrm{~mm})\end{array}$ & $p^{\prime}$ & $C(\mathrm{kPa})$ & $\tan \varphi\left(^{\circ}\right)$ & $\begin{array}{l}\text { 黏粒 } \\
\text { 含量 }\end{array}$ & $\begin{array}{c}d_{50} \\
(\mathrm{~mm})\end{array}$ & $p^{\prime}$ & $C(\mathrm{kPa})$ & $\tan \varphi\left(^{\circ}\right)$ \\
\hline 1 & 板桥 & 23.0 & 3.0 & 4.0 & 4.0 & 0.03 & 0.3 & 30.0 & 0.5 & 0.4 & 0.2 & 0.35 & 0.0 & 0.37 \\
\hline 2 & Castlewood & 20.0 & 4.0 & 4.0 & 4.0 & 0.3 & 0.22 & 15.0 & 0.5 & 0.2 & 100 & 0.35 & 0.0 & 0.9 \\
\hline 3 & Coedty & 10.0 & 2.0 & 4.0 & 4.0 & 0.03 & 0.3 & 30.0 & 0.5 & 0.4 & 0.2 & 0.35 & 0.0 & 0.37 \\
\hline 4 & Elk City & 8.5 & 2.0 & 4.0 & 4.0 & 0.03 & 0.3 & 30.0 & 0.5 & 0.4 & 0.2 & 0.35 & 0.0 & 0.37 \\
\hline 5 & Grand Rapids & 7.0 & 2.0 & 4.0 & 4.0 & 0.03 & 0.3 & 30.0 & 0.5 & 0.4 & 0.2 & 0.35 & 0.0 & 0.37 \\
\hline 6 & $\begin{array}{c}\text { IMPACT现 } \\
\text { 场试验 }\end{array}$ & 5.3 & 1.5 & 4.0 & 4.0 & 7.0 & 0.24 & 20.0 & 1.0 & 0.0 & 85.0 & 0.24 & 0.4 & 0.9 \\
\hline 7 & 刘家台 & 28.0 & 20.0 & 0.333 & 2.0 & 0.03 & 0.3 & 30.0 & 0.5 & 0.4 & 0.2 & 0.35 & 0.0 & 0.37 \\
\hline 8 & Oros & 35.4 & 5.0 & 0.9 & 0.45 & 0.2 & 0.35 & 41.2 & 0.51 & 0.1 & 2.5 & 0.28 & 0.0 & 0.85 \\
\hline 9 & 竹沟 & 23.0 & 3.0 & 4.0 & 4.0 & 0.03 & 0.3 & 30.0 & 0.5 & 0.4 & 0.2 & 0.35 & 0.0 & 0.37 \\
\hline 10 & 佐村 & 25.0 & 3.0 & 4.0 & 4.0 & 0.03 & 0.3 & 30.0 & 0.5 & 0.4 & 0.2 & 0.35 & 0.0 & 0.37 \\
\hline
\end{tabular}

表 3 黏土心墙坝漫顶溃坝案例模型实测值与计算结果比较

\begin{tabular}{|c|c|c|c|c|c|c|c|c|c|}
\hline \multirow{2}{*}{ 序号 } & \multirow{2}{*}{ 大坝名称 } & \multicolumn{4}{|c|}{ 实测值 } & \multicolumn{4}{|c|}{ 计算结果 } \\
\hline & & $Q_{p}\left(\mathrm{~m}^{3} / \mathrm{s}\right)$ & $B_{t}(\mathrm{~m})$ & $B_{b}(\mathrm{~m})$ & $T_{p}\left(T_{f}\right)(\mathrm{h})$ & $Q_{p}\left(\mathrm{~m}^{3} / \mathrm{s}\right)$ & $B_{t}(\mathrm{~m})$ & $B_{b}(\mathrm{~m})$ & $T_{p}\left(T_{f}\right)(\mathrm{h})$ \\
\hline 1 & 板桥 & 78100 & 372.0 & 210.0 & $-(5.5)$ & 74670.8 & 439.6 & 241.4 & $1.42(4.51)$ \\
\hline 2 & Castlewood & 3570 & 54.9 & 33.5 & $-(-)$ & 3309.7 & 86.3 & 37.4 & $1.13(1.37)$ \\
\hline 3 & Coedty & - & 67.0 & 18.2 & $-(-)$ & 334.8 & 48.4 & 26.4 & $0.99(2.39)$ \\
\hline 4 & Elk City & - & 45.5 & 27.7 & $-(0.83)$ & 800.8 & 59.2 & 29.6 & $0.76(0.85)$ \\
\hline 5 & Grand Rapids & - & 12.2 & 9.1 & $-(0.5)$ & 372.9 & 14.6 & 11.7 & $0.36(0.55)$ \\
\hline 6 & IMPACT现场试验 & 242 & - & - & & 229.5 & 22.7 & 18.1 & $5.17(5.27)$ \\
\hline 7 & 刘家台 & 28000 & - & - & $-(-)$ & 30096.7 & 311.7 & 171.0 & $0.77(6.50)$ \\
\hline 8 & Oros & $\begin{array}{c}12000 \sim \\
58000\end{array}$ & 200.0 & 130.0 & $-(-)$ & 51191.1 & 249.2 & 170.4 & $4.66(6.36)$ \\
\hline 9 & 竹沟 & 11200 & 159.0 & 110.0 & $-(0.43)$ & 9627.1 & 137.5 & 90.2 & $0.34(0.43)$ \\
\hline 10 & 佐村 & 23600 & - & - & $-(1.0)$ & 26308.3 & 268.4 & 160.2 & $0.79(1.43)$ \\
\hline
\end{tabular}

表 4 模型各参数计算结果误差统计

\begin{tabular}{ccccc}
\hline & $Q_{p}$ & $B_{t}$ & $B_{b}$ & $T_{f}$ \\
\hline 有实测结果案例数 & 7 & 7 & 7 & 5 \\
相对误差 $\pm 25 \%$ 以内案例的比例 & $100 \%$ & $57.1 \%$ & $57.1 \%$ & $80.0 \%$ \\
相对误差 $\pm 50 \%$ 以内案例的比例 & $100 \%$ & $85.7 \%$ & $100 \%$ & $100 \%$ \\
均方根相对误差 & 0.042 & 0.117 & 0.097 & 0.082 \\
\hline
\end{tabular}

通过表3和表4发现, 溃口峰值流量的计算误差较 小, 溃口最终宽度和溃坝历时的计算误差较大, 这主 要与坝料性质的复杂性和溃坝历时的统计有关, 对于 年代久远的大坝, 坝料的实测资料较少, 因此常采用
假设的参数; 而对于溃坝历时, 往往是在溃坝以后通 过反馈分析或者实地调查获取, 也可能存在较大误差. 但通过比较 10 个溃坝案例的计算值与实测值发现, 本 文建立的模型可较好地模拟黏土心墙坝漫顶溃坝过 
程, 计算结果也可满足工程需求.

\section{5 结论}

基于溃坝模型揭示的黏土心墙坝漫顶溃决机理, 建立了一个可模拟黏土心墙坝漫顶溃坝过程的数学 模型, 并提出了相应的数值计算方法. 选择国内外 10
个具有实测数据的黏土心墙坝漫顶溃坝案例来验证 模型的合理性, 通过比较计算值与实测值发现, 本文 模型可较好地反映溃口峰值流量, 溃口最终宽度与溃 坝历时等重要参量, 并可通过每个时间步长溃口洪水 流量的计算, 得到溃口流量过程线, 为溃坝洪水演进 的模拟提供边界条件, 为黏土心墙坝漫顶溃坝致灾后 果的评价提供理论与技术支撑.

\section{参考文献}

1 周建平, 王浩, 陈祖煜, 等. 特高坝及其梯级水库群设计安全标准研究I: 理论基础和等级标准. 水利学报, 2015, 46: 505-514

2 Zhang H, Chen J K, Hu S W, et al. Deformation characteristics and control techniques at the Shiziping earth core rockfill dam. J Geotech Geoenviron Eng, 2016, 142: 04015069

3 水利部大坝安全管理中心. 全国水库垮坝登记册. 南京: 水利部大坝安全管理中心, 2015

4 汝乃华, 牛运光. 大坝事故与安全·土石坝. 北京: 中国水利水电出版社, 2001

5 陈生水. 土石坝溃决机理与溃决过程模拟. 北京: 中国水利水电出版社, 2012

6 ASCE/EWRI Task Committee on Dam/Levee Breaching. Earthen embankment breaching. J Hydraul Eng, 2011, 137: 1549-1564

7 Zhong Q M, Wu W M, Chen S S, et al. Comparison of simplified physically based dam breach models. Nat Hazards, 2016, 84: 1385-1418

8 钟启明, 陈生水, 邓㖣. 均质土坝漫顶溃坝过程数学模型研究及应用. 水利学报, 2016, 47: 1519-1527

9 Morris M W. IMPACT Project Field Tests Data Analysis. Oxfordshire: HR Wallingford, 2008

10 陈生水, 钟启明, 曹伟. 黏土心墙坝漫顶溃决过程离心模型试验与数值模拟. 水科学进展, 2011, 22: 87-92

11 Singh V P. Dam Breach Modeling Technology. Dordrecht: Kluwer Academic Publishes, 1996

12 Fread D L. DAMBREAK: The NWS Dam Break Flood Forecasting Model. Silver Spring: National Oceanic and Atmospheric Administration, 1984

13 Visser P J. Breach Growth in Sand-Dikes. Delft: Delft University of Technology, 1998

14 Wu W M. Simplified physically based model of earthen embankment breaching. J Hydraul Eng, 2013, 139: 837-851

15 US Department of Agriculture. Natural Resources Conservation Service (USDA-NRCS), Chapter 51: Earth spillway erosion model, Part 628 Dams. Washington DC: National Engineering Handbook, 1997

16 Briaud J L, Ting F C K, Chen H C, et al. Erosion function apparatus for scour rate predictions. J Geotech Geoenviron, 2001, 127: 105-113

17 Wan C F, Fell R. Investigation of rate of erosion of soils in embankment dams. J Geotech Geoenviron, 2004, 130: 373-380

18 Temple D M, Hanson G J. Headcut development in vegetated earth spillways. Appl Eng Agric, 1994, 10: 677-682

19 Wu W M. Computational River Dynamics. London: Taylor \& Francis, 2007

20 Temple D M. Estimating flood damage to vegetated deep soil spillways. Appl Eng Agric, 1992, 8: 237-242

21 梅世昂, 霍家平, 钟启明. 均质土坝漫顶溃决“陡坎”移动参数确定. 水利水运工程学报, 2016, 2: 24-31

22 Morris M W, Hassan M, Vaskinn K A. Breach Formation Technical Report (WP2). Oxfordshire: HR Wallingford, 2005

23 Wahl T L. Prediction of embankment dam breach parameters: A literature review and needs assessment. Denver: Bureau of Reclamation, 1998

24 Xu Y, Zhang L M. Breaching parameters for earth and rockfill dams. J Geotech Geoenviron, 2009, 135: 1957-1970 


\title{
Numerical model for clay core dam breach process due to overtopping considering different failure modes of core wall
}

\author{
ZHONG QiMing ${ }^{1,2}$, CHEN ShengShui ${ }^{1,2}$, CAO Wei ${ }^{1} \&$ MEI ShiAng ${ }^{1}$ \\ ${ }^{1}$ Nanjing Hydraulic Research Institute, Nanjing 210029, China; \\ ${ }^{2}$ Key Laboratory of Earth-Rock Dam Failure Mechanism and Safety Control Techniques, Ministry of Water Resources, Nanjing 210029, China
}

Based on the breach mechanism of clay core dam due to overtopping failure, a numerical model for dam breaching process is put forward. In this model, the initial scour position on the downstream slope is determined by means of the characteristics of dam figuration and overtopping flow, the broad-crested weir formula is adopted to calculate breach flow discharge, and a time averaged backward erosion formula is adopted to simulate the dam shell breach. The moment and mechanical equilibrium methods are utilized to model the overturning and sliding failures of the core, respectively. In order to simulate the actual failure mode of the dam, one-and two-sided erosion, as well as dam base erosion, are taken into account. The model adopts the iterative calculation method to simulate the coupling process of water and soil during dam breaching. 10 clay core dam overtopping failure cases which have measured data are adopted to testify the present model. The comparison of measured data and calculated results on peak breach flow, final breach width and failure time shows that the calculated results can meet the engineering requirements, and the statistics of model's relative errors validate the reasonability of the present model.

clay core dam, overtopping, core wall failure, numerical model

doi: 10.1360/N092017-00135 